\title{
Carlos Fernández Casado y José Acuña: los primeros puentes de altura estricta. Jaén, 1933-1935
}

\author{
Carlos Fernández Casado and José Acuña: \\ The first "strict height" bridges. Jaén, 1933-1935 \\ $\underline{\text { A. Burgos }}^{(*)}$, M.P. Saéz-Pérez(*), J.C. Olmo(*)
}

RESUMEN

La "Colección de Puentes de Altura Estricta" fue una de las mayores creaciones del genial ingeniero de caminos español Carlos Fernández Casado. Fue diseñada a principios de los años 1930 y tuvo una gran trascendencia en la Ingeniería civil española del siglo XX. No obstante, sus orígenes son poco conocidos.

Las primeras materializaciones de la "Colección" fueron seis puentes que se construyeron en la provincia de Jaén, entre 1933 y 1935 . Fueron posibles gracias al decidido apoyo que prestó otro ingeniero de caminos, José Acuña y Gómez de la Torre. Posteriormente también se hizo cargo de su ejecución material.

Con esta investigación se pretende reconstruir su proceso de diseño y construcción. También se describen los puentes tal como han llegado hasta nuestros días. Con todo ello se espera contribuir a que sea reconocido su gran valor como elementos patrimoniales.

$560-37$

Palabras clave: Ingeniería; puentes de altura estricta; hormigón armado; Jaén; patrimonio.

\section{SUMMARY}

The bridge design catalogue "Puentes de altura Estricta" (Strict Height Bridges) was one of the greatest creations of the brilliant spanish civil engineer Carlos Fernandez Casado. It was designed at the early 1930's. It had a great relevance in the Spanish Civil Engineering of the 20th century. Nevertheless, its origins are little known.

The first materializations of the "Collection" were six bridges that were constructed in the Jaén province, between 1933 and 1935. They were possible thanks to the determined support of other civil engineer, Jose Acuña y Gómez de la Torre. Later also he carried out the work himself.

With this research its process of design and construction is reconstructed. Also bridges are described as they have come to the present day. With all this it is expected its great value as heritage elements be recognized.

Keywords: Engineering; bridges of strict height; reinforced concrete; Jaén; heritage.

\footnotetext{
(*) Escuela Técnica Superior de Ingeniería de Edificación, Universidad de Granada (España).
} Persona de contacto/Corresponding author: abn@ugr.es (A. Burgos) 


\section{INTRODUCCIÓN}

Carlos Fernández Casado ocupa un lugar muy destacado dentro de la Ingeniería Civil española del siglo XX. Fue uno de nuestros grandes ingenieros estructurales, tanto en el plano de la Construcción propiamente dicha como en el de la Teoría y la Producción Científica, campos ambos en los que realizó aportaciones numerosas y sobresalientes. Pero además, como intelectual polifacético, trascendió de las cuestiones puramente técnicas para profundizar sobre el sentido de la Arquitectura y la Ingeniería a partir de reflexiones surgidas desde la perspectiva de la Historia y la Filosofía.

En lo relativo a la dimensión puramente ingenieril de su Obra, uno de sus principales logros, fue la "Colección de puentes de altura estricta", un conjunto de diseños estandarizados, magníficamente determinados desde todos los puntos de vista (estructural, constructivo, funcional y estético), que puso a disposición de todos sus compañeros ingenieros para la resolución del problema del proyecto de puentes comunes para carreteras y líneas de ferrocarril.

Según (1) la "Colección" estuvo vigente desde 1933 a 1955. En un primer periodo inmediatamente anterior a la Guerra Civil se hicieron seis puentes (más dos pequeños pasos superiores), en cuyo proyecto y construcción participaría de forma muy activa Carlos Fernández Casado. En Jaén se localizaron cuatro puentes y las obras de paso, siendo los dos restantes, los famosos puentes de Puerta de Hierro y de El Pardo, que se construyeron en los alrededores de Madrid.

Finalizada la contienda, la "Colección" fue aprobada oficialmente mediante Orden Ministerial de 22 de diciembre de 1939 (2), siendo sus modelos aplicados con cierta frecuencia en la construcción de puentes en la década siguiente por todo el territorio nacional. Hasta el final del periodo en 1955, cuando la difusión de la técnica del hormigón pretensado la dejara obsoleta, se hicieron alrededor de cincuenta aplicaciones de la "Colección", las más significativas, obras del propio Fernández Casado. Concretamente en Jaén, según consta en (1) durante todo el periodo se realizaron un total de 8 puentes (los seis de antes de la Guerra Civil y dos nuevos pasos superiores)

Así pues, los primeros ejemplares de la "Colección" se materializaron en la provincia de Jaén entre 1933 y 1934. Fueron tres puentes de carretera por los que se atrevió a apostar el ingeniero de caminos José Acosta, por entonces responsable de buen número de estas infraestructuras en la provincia, que primero los incluyó en sus propios proyectos y después llevaría a cabo efectivamente su construcción. De esta afortunada colaboración surgirían posteriormente (1935) otro puente más y dos pasos superiores.

El ingeniero León García Merino construyó hacia 1945 dos pequeños pasos superiores de ferrocarril junto a las localidades de Mengibar y Villargordo (3).

La obra de Fernández Casado y en particular la "Colección de puentes de altura estricta" ha sido estudiada con cierta profundidad. Sus ejemplares más representativos han sido objeto de atención, para empezar por parte del propio autor, en diversos artículos y monografías. Sin embargo, de sus primeras materializaciones, aparte de algunas referencias de los comienzos de la "Colección", apenas se tenía conocimiento hasta ahora (4).

El descubrimiento de documentos inéditos en el Archivo Histórico Provincial de Jaén nos ha permitido acceder a nueva información sobre su proceso de diseño y construcción.

Concretamente se ha contado con distintos planos y documentos administrativos (actas de recepción, mediciones y correspondencia entre el personal de la División Provincial de Obras Públicas de Jaén) que en los distintos apartados del presente artículo se irán exponiendo.

Por otra parte y complementariamente al estudio de las referencias bibliográficas, se ha llevado a cabo un minucioso trabajo de campo en el que se ha procedido a su localización, reconocimiento y definición del estado de conservación, quedando constancia de ello en los siguientes apartados.

En la actualidad se conservan solamente tres de estos puentes, aunque están tristemente olvidados y sin que nadie parezca reconocer su valor. No obstante, su magnífica factura les permite continuar, pese a todo, en pie y prestando servicio.

Este artículo pretende dar a conocer su existencia y poner de relieve su gran importancia y valor histórico. Con su conocimiento, los autores esperamos poder contribuir a la recuperación de estas magníficas construcciones y a su justa integración en el Patrimonio de la Arquitectura y la Ingeniería de nuestro país. 


\section{LA COLECCIÓN DE PUENTES DE ALTURA ESTRICTA. ANTECEDENTES Y CONTEXTUALIZACIÓN}

El origen de la "Colección de puentes de altura estricta" está relacionado con los inicios del hormigón armado y las nuevas necesidades en la construcción de puentes de carretera en el primer tercio del siglo XX en España.

La "Colección" apareció en el periodo de generalización del hormigón armado en nuestro país. De hecho, podría considerarse como uno de los hitos que señalan inequívocamente la consolidación "de la nueva técnica constructiva".

Hasta entonces, la construcción metálica había acaparado el mayor protagonismo en el ámbito de la Arquitectura y de la Ingeniería. El hierro era el material estructural preferido por los técnicos, no cuestionándose su empleo en las obras relevantes, como los grandes puentes y viaductos de las líneas ferroviarias o la estructura de entramados de los principales edificios.

En lo relativo a los puentes, la materialización de la red de ferrocarril, en la segunda mitad del siglo XIX, sería determinante para la preponderancia de la construcción metálica. De igual forma también tendría gran trascendencia en la formación de la red principal de carreteras, diseñada en base a los planes generales de 1860 y 1877 . En ambos tipos de infraestructuras los puentes de cierta entidad se hicieron metálicos, de muy variadas tipologías, aunque predominando las vigas trianguladas rectas.

No obstante, y particularmente en las carreteras, los puentes de luces medianas y reducidas continuaron resolviéndose mayoritariamente con los tradicionales arcos de fábrica.

A partir de 1910, materializada prácticamente en su totalidad la red principal, se empezaría a estimular la construcción de las carreteras de tercer orden, provinciales y vecinales. La Administración de la Dictadura de Primo de Rivera asumiría su desarrollo como objetivo preferente, junto con la creación de un conjunto especial de carreteras seleccionadas de gran calidad (el famoso Circuito Nacional de Firmes Especiales). Durante la II República se siguió impulsando con fuerza la construcción de carreteras vecinales, que en muchos casos serían terminadas después de la Guerra Civil (5).

Estas nuevas prioridades en las carreteras modificaron las necesidades de los ingenieros encargados de su diseño y construcción. Si bien no dejaron de hacer falta todavía bastantes puentes medianos y de gran tamaño, lo más apremiante pasaría a ser la construcción de un ingente número de obras pequeñas, destinadas a vías de tráfico reducido.

En estas circunstancias, la Administración consideró oportuno poner, a disposición de los ingenieros encargados del proyecto de estas carreteras, un repertorio estandarizado de puentes sencillos (6). Con el precedente de la de tajeas y obras de paso de 1859, que había resultado de gran utilidad, hacia 1915 se comenzó a promover la redacción de una colección oficial de puentes económicos.

El impulso cristalizaría en los años 1920 con la publicación de varias series de puentes de hormigón armado: las dos primeras (1919 y 1921), de tramos rectos debidas a Juan Manuel de Zafra; y la tercera (1923), de puentes arco ideada por José Eugenio Ribera (7).

Posteriormente saldría a la luz otra colección similar de puentes metálicos (redactada por el ingeniero Domingo Mendizábal). Pero a esas alturas ya estaba claro que el hormigón armado iba a ser el material estructural básico en el diseño de estas nuevas infraestructuras.

Tal y como se ha comentado el hormigón armado se introdujo en España en torno a 1900. En los primeros años su utilización estuvo condicionada por patentes y privilegios comerciales. Pero a partir de 1911 la formulación racional de su base teórica, realizada en nuestro país principalmente por Juan Manuel de Zafra, posibilitó que fuera usado libremente y sin cortapisas comerciales. Desde ese momento, sucesivas generaciones de técnicos, formados ya en la nueva práctica constructiva, impulsarían decididamente su desarrollo, sobre todo en el ámbito de la Ingeniería Civil y particularmente en la construcción de puentes.

Ante las nuevas necesidades de puentes pequeños, el hormigón armado se reveló como la técnica más ventajosa. El nuevo material permitía la implantación de la tipología de viga recta (que permitía una mayor sección de desagüe) para pequeñas luces, imposible de hacer con las fábricas tradicionales e inviable por antieconómica si se realizaba con elementos metálicos.

Además, a principios de los años 1920 ya se habían superado los recelos despertados inicialmente por el hormigón armado (al menos en el ámbito de la Ingeniería). Los puentes construidos con el nuevo material ya habían demostrado suficientemente su aptitud, siendo además mucho más baratos y fáciles de construir 
1. Sección transversal genérica de los dos tipos de tableros en los puentes de altura estricta (4).
Las colecciones de modelos oficiales contribuirían decisivamente a su generalización. Los diseños de puentes arco de Ribera serían los más empleados en luces medianas y grandes. En cambio, para luces reducidas los tramos rectos con sección " $\pi$ " de Zafra tuvieron una rápida y amplia aceptación. En pocos años, las carreteras de toda la geografía nacional se llenarían de este tipo de puentes.

\subsection{Carlos Fernández Casado en Andalucía Oriental. Los comienzos de su trayectoria profesional}

El destacado papel de Carlos Fernández Casado en la ingeniería civil española en el S. XX permite que se reconozca como una de las personalidades más brillantes tanto por su actividad como ingeniero como por su vertiente de humanista versado en materias como la Historia y la Filosofía. De hecho fue justamente reconocido en vida como "cima inalcanzable" de la profesión (8). En toda su producción, tan amplia como diversa, dejó constancia de su constante reflexión sobre el sentido y la trascendencia de la obra de ingeniería constituyendo sus realizaciones valiosos ejemplos del diálogo entre la más alta competencia técnica, la sensibilidad hacia la naturaleza y una permanente búsqueda de la belleza.

Su obra en conjunto constituye una contribución tan relevante a la Ingeniería española del siglo $X X$, que es muy difícil destacar alguna de sus realizaciones. De pretenderlo, tal vez habría que elegir su "Colección de puentes de altura estricta", aunque sólo fuese por ser, según propia confesión, "de lo que más contento estaba de haber hecho" (9).

A pesar de que sólo tenía 28 años cuando la diseñó, la "Colección" no fue ni mucho menos uno de sus primeros trabajos. Fernández Casado (Logroño, 1905), tras completar sus estudios de ingeniero de Caminos (1924), de Radiotelegrafía (1925) y de Telecomunicaciones (1927), fue a establecerse a Granada. Allí, además de tomar parte en la por entonces animada y vanguardista vida cultural de la ciudad, empezó a ejercer la primera de esas tres profesiones.

Sus primeras realizaciones serían caminos vecinales, canales de riego y pequeños saltos de agua, que llevaría a cabo por toda la provincia, así como en las limítrofes de Jaén y Almería. De esta forma transcurrieron los primeros cuatro años de su carrera profesional, plenos de experiencias y muy satisfactorios a nivel personal. Sin embargo, al final de este periodo experimentó una crisis que le hizo reflexionar sobre lo que había realizado hasta ese momento.
Sintiendo que no había llevado a cabo ninguna obra significativa, decidió dar un giro a su carrera, proponiéndose la realización de trabajos verdaderamente trascendentes.

Fue entonces, poco antes de su regreso a Madrid (que tuvo lugar en 1932), cuando concibió la idea de la "Colección de puentes de altura estricta". No tardaría demasiado en completarla, cosa que hizo mientras trabajaba en diversos proyectos e iniciaba su colaboración con la empresa constructora Huarte.

\section{LA COLECCIÓN DE PUENTES DE ALTURA ESTRICTA. ASPECTOS CONSTRUCTIVOS Y FUNCIONALES. PREMISAS BÁSICAS DEL DISEÑO}

\subsection{Características generales de la "Colección"}

En una primera instancia, el objetivo de Fernández Casado al concebir, en 1933, los puentes de altura estricta fue resolver el "paso de cauces corrientes por carreteras de mediana importancia", problema que él mismo había tenido que afrontar en numerosas ocasiones en los comienzos de su andadura profesional (10).

Para ello no se planteaba ya más alternativa que la aplicación de alguno de los diseños de la "Colección" de modelos oficiales de tramos rectos de hormigón armado. Sin embargo, en muchas ocasiones esto conducía a tableros de canto relativamente grande, que, o bien obligaban a elevar la rasante de la carretera y consecuentemente aumentar la obra de acompañamiento (terraplenes, estribos, muros,...), o bien reducían la sección de desagüe.

Ante tal situación, Fernández Casado asumió el reto de diseñar una solución más eficiente, empezando su trabajo por la trascendental cuestión de la forma estructural. Tras un complejo análisis del estado de la cuestión en otros países, se decidió por la solución de tablero superior, formado por una losa o por vigas de sección transversal en " $\mathrm{T}$ ".

Por otra parte, junto a la opción de losa, ventajosa por su sencillez de ejecución y economía, contempló la posibilidad de construir tableros con vigas para el caso de luces importantes. Ambas soluciones se muestran en la Figura 1.

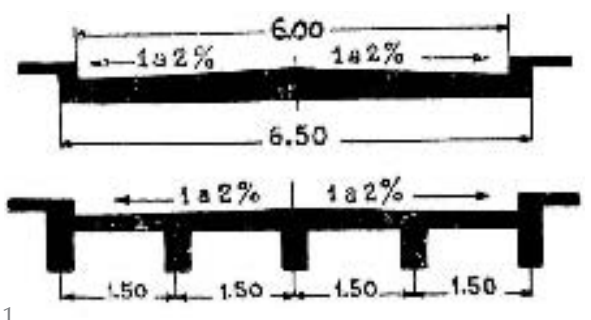


En sentido longitudinal proponía cuatro disposiciones básicas, las tres primeras hiperestáticas: el pórtico sencillo, el pórtico de tres vanos sin pilares extremos (sección "TT") y el dintel de tres vanos sobre cuatro apoyos simples; de esta última, como cuarta posibilidad, se ofrecía la versión isostática con el tramo central en cantiléver. Estaban formadas por tramos individuales de hasta 30 metros de luz y como signo distintivo, todas ellas mostraban acartelamientos en sentido longitudinal, según el perfil de igual resistencia a flexión. Las diferentes combinaciones según las clasificó el propio Fernández Casado se muestran en la Figura 2.

Es importante destacar que estas configuraciones fueron el resultado de un exhaustivo análisis estructural y de optimización de la ejecución de diferentes alternativas.

Para su cálculo en la primera disposición (pórtico sencillo) Fernández Casado utilizó como herramienta básica el método de Hardy Cross, que él mismo había introducido en España (12).

Combinando estas configuraciones básicas y alternando entre tableros de losa y de vigas, resultaban hasta diez series de puentes, con las que se podía dar respuesta a las situaciones más diversas (incluyendo el cruce con otras carreteras y líneas de ferrocarril).

Aunque en algunos casos se resolvieron con palizadas, como solución típica para los apoyos (intermedios y estribos) se recurrió a tabiques de espesor constante (biselados en las pilas para formar tajamares triangulares).

Las cuestiones hidráulicas se tuvieron también muy en cuenta en la definición del diseño final. En este sentido, uno de los planteamientos más originales fue contemplar la posibilidad de mantener los puentes completamente sumergidos. Esta fue una de las principales razones por las que se adoptó preferentemente la solución de losa como tablero en los puentes de menor entidad, ya que era la que ofrecía el menor obstáculo al desagüe.

Consideraciones de esta naturaleza resultaban decisivas por lo general en la elección de la configuración estructural. Por ejemplo, la de pórtico en $\mathrm{p}$ se recomendaba para paso de arroyos torrenciales, pues era muy resistente al choque de grandes cantos. En cambio, las series VII y VIII estaban especialmente indicadas para terrenos de cimentación dudosos, pues por su condición de isostáticas sufrían menos en caso de asiento de los apoyos.

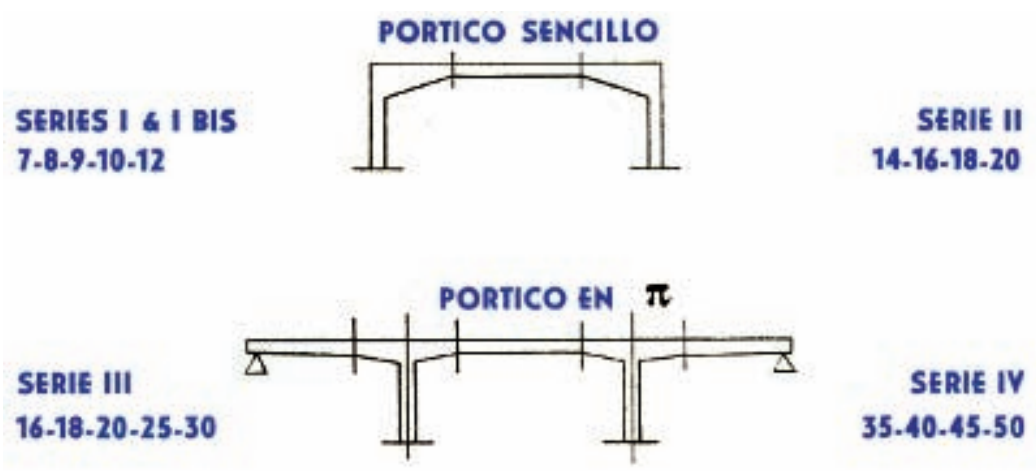

SERIE $V$

16.18-20-25-30

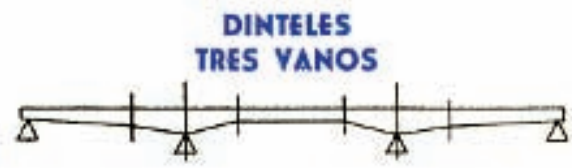

SERIE VI 35.40.45.50

\section{8-20-25-35.40}

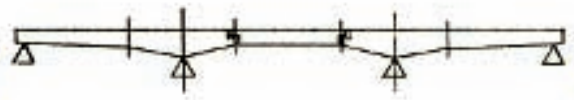

SERIE VIII 45.50-55.65.75

Por último, se perfiló el diseño de los puentes de altura estricta para que pudieran ser construidos fácilmente y con los mínimos medios auxiliares. Fernández Casado dispuso dos procedimientos para la colocación en obra de la armadura, contemplando la posibilidad de ensamblar previamente los cuchillos. Los encofrados eran todos muy sencillos y el proceso general de construcción del tablero a partir de las pilas-tabique simplificaba extraordinariamente las cimbras. Una muestra de la idoneidad de este diseño puede reconocerse en la cimbra y encofrados que se utilizaron en el puente sobre el río Guadalimar en La Puerta de Segura, que el propio ingeniero fotografió durante su construcción (Figura 3).

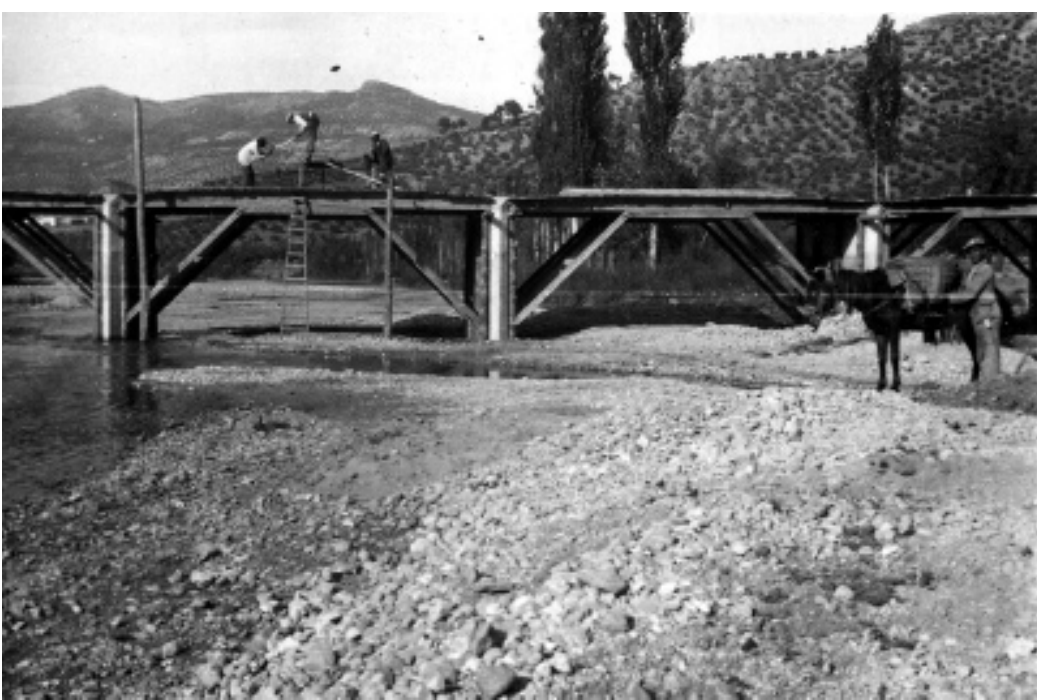

2. Configuraciones estructurales de los puentes de altura estricta (11)

3. Construcción del puente sobre el río Guadalimar en La Puerta de Segura (Jaén). Carretera de 3er orden de la Puerta de Segura a Puente Génave, 1935 (13). 
4. Proyecto de puente sobre el río de la Vega en Santo Tomé para la carretera de 3er orden de Peal de Becerro a Villacarrillo. 1932. Plano de alzado general (19).
Los puentes de altura estricta, por tanto, fueron concebidos con la mayor brillantez desde el punto de vista técnico. Pero no sólo las cuestiones funcionales habían intervenido en su formulación. Desde la personal concepción que su creador tenía del sentido y trascendencia de las obras de ingeniería, la estética, debía ser siempre un capítulo fundamental de su diseño.

Así, para Fernández Casado al proyectar una obra era necesario tener presente, lógicamente en primer lugar, satisfacer las necesidades que habían determinado su construcción. Pero además había que conseguirlo aunando racionalidad técnica, economía y belleza.

En el caso de los puentes de altura estricta, para alcanzar la belleza la norma básica era que el puente no trastornara la armonía del lugar donde se construía. Nadie lo podría explicar mejor que el propio Fernández Casado al revelar como justificación última de la estética de sus puentes su aspiración de "introducir el mínimo de ideas nuevas sobre el paisaje, tan sólo un contorno sencillo que pudiera dibujarse sobre la nieve" (1).

\section{LOS PUENTES DE ALTURA ESTRICTA, CONSTRUIDOS EN LA PROVINCIA DE JAÉN}

\subsection{Circunstancias que dieron lugar a la construcción de los primeros puentes de la Colección en Jaén}

En el arranque de la "Colección de puentes de altura estricta" tendría gran trascendencia la intervención de José Acuña y Gómez de la Torre, como el propio Fernández Casado no dejaría de reconocer al considerarle el padrino de la "Colección" (14).

Este singular y polifacético ingeniero de caminos (fue además inventor y político) trabajaba desde 1927 en la Jefatura provincial de Obras Públicas de Jaén, donde era responsable del proyecto y construcción de los caminos vecinales (15).

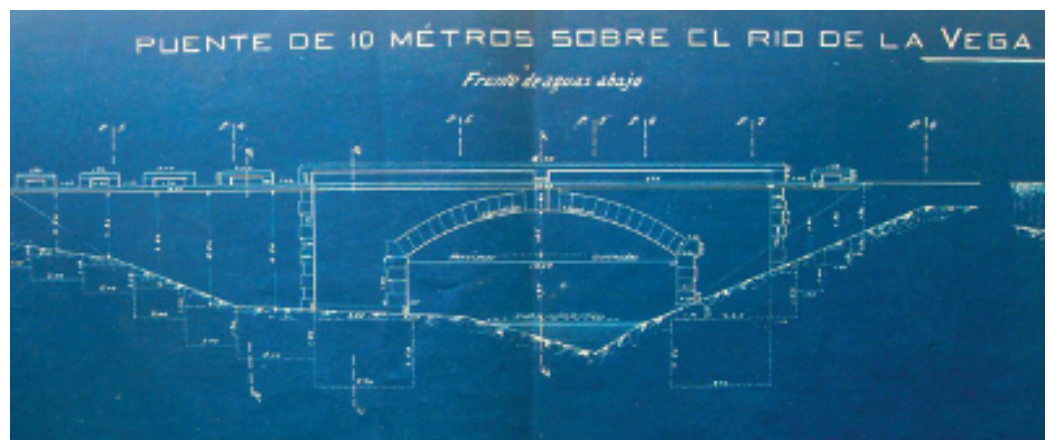

En el momento de su incorporación, las infraestructuras y más concretamente, la red provincial de estos caminos secundarios, estaba muy poco desarrollada, acumulando algunos de ellos varias décadas de retraso en su ejecución.

Un ejemplo de lo comentado, según consta en (16), se encuentra en la carretera de tercer orden de Peal de Becerro a Villacarrillo por Santo Tomé y Mogón, cuyo proyecto inicial databa de 1887. Dividida en cuatro tramos, todavía en 1931 estaban sin ejecutar los dos últimos.

A esta circunstancia se añadía la aguda crisis económica y social que atravesaba la zona (puesta de manifiesto por múltiples testimonios de la época), que indujo a plantear la terminación de la carretera como único medio de dar trabajo a la multitud de trabajadores agrícolas desempleados. Desde algunos ayuntamientos se solicitó al Ministerio de Fomento, en aras de evitar un grave conflicto, la ejecución inmediata y por administración de las obras (17).

\subsection{Puente sobre el río de la Vega en Santo Tomé, 1933}

El Ministerio cedió ante estos apremios, encargándose a José Acuña la redacción urgente de un anteproyecto o replanteo previo, con el que pudieran empezar los trabajos. El proyecto (18) presentado en abril de 1932, comprendía un trazado de $14 \mathrm{~km}$, entre los que se incluía como obra más relevante un puente de $10 \mathrm{~m}$ de luz sobre el río de la Vega, en las inmediaciones de la localidad de Santo Tomé.

Si bien Acuña definió con solvencia las condiciones generales de la explanación y las pequeñas obras accesorias, por el contrario para el puente incluyó la ya por entonces anacrónica solución de arco escarzano de fábrica, prolongado por muros de acompañamiento. En la Figura 4 se muestra lo comentado.

El propio Acuña era consciente de lo inadecuado de tal propuesta y cuando llegó la hora efectiva de la ejecución, decidió recurrir a Carlos Fernández Casado.

Así empezaría una fructífera colaboración entre ambos ingenieros, que tal vez se remontara a algunos años atrás, a finales de los años veinte. Acuña gestionaba por esa época un salto hidroeléctrico en la comarca de la Sierra Sur de Jaén, siendo muy posible que coincidiera allí con Fernández Casado, que por entonces hacía por la zona trabajos como el proyecto de un salto de agua y un canal de riego en el río Víboras, dentro del término municipal de Fuensanta de Martos (18). 
Desde todos los puntos de vista Fernández Casado resolvió airosamente el paso de la carretera sobre el río de la Vega con el primer puente de altura estricta, un modelo de la serie III (pórtico en p y tablero de losa) con tres vanos de luces 5,70-7,60-5,70 m. Estaba apoyado sobre estribos y dos esbeltas pilas-tabique de 5,70 m de altura (Figura 5).

Su configuración estructural y detalles responden al diseño general de la "Colección" descrito por el autor en diversas publicaciones (20) y contemplado posteriormente en la disposición ministerial de 1942 en la que se aprobó oficialmente la "Colección" (21).

Su ejecución se llevó a cabo a lo largo de 1933, siendo su contratista el empresario jiennense Juan Delgado García. En su construcción se debieron cometer algunas irregularidades administrativas, ya que en la primera acta de recepción se consignó la construcción de un puente de fábrica de $10 \mathrm{~m}$ de luz. Esto fue corregido en el acta definitiva, firmada en enero de 1936 (23).

En la Figura 6 se muestra una vista del puente durante su construcción. Puede constatarse que aún no habían sido depurado del todo algunos aspectos del diseño, como es el caso de las cimbras que aquí son más rudimentarias que en el puente de La Puerta de Segura anteriormente citado.

No obstante, no cabe duda de que el puente quedó construido a total satisfacción, pues acto seguido Acuña encargó a Fernández Casado otros dos más de la colección de altura estricta para otra carretera de su competencia.

\subsection{Puentes de la carretera de La Puerta de Segura a Siles, 1934}

Se trataba en este caso de la carretera de tercer orden de la Puerta de Segura a Siles, cuya construcción, también a petición de los ayuntamientos implicados, fue decidida en 1929. Inmediatamente fue encargado su proyecto a José Acuña, que lo presentó a la Superioridad en diciembre de ese mismo año (25).

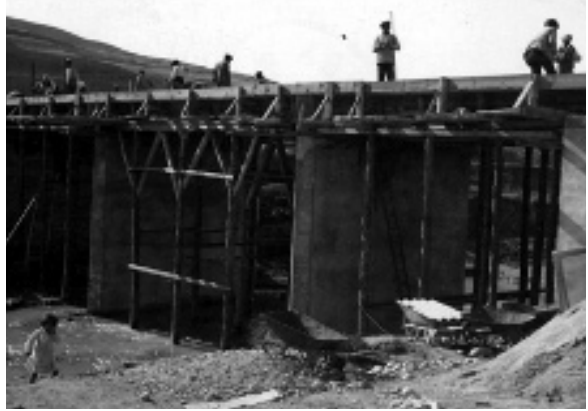

6

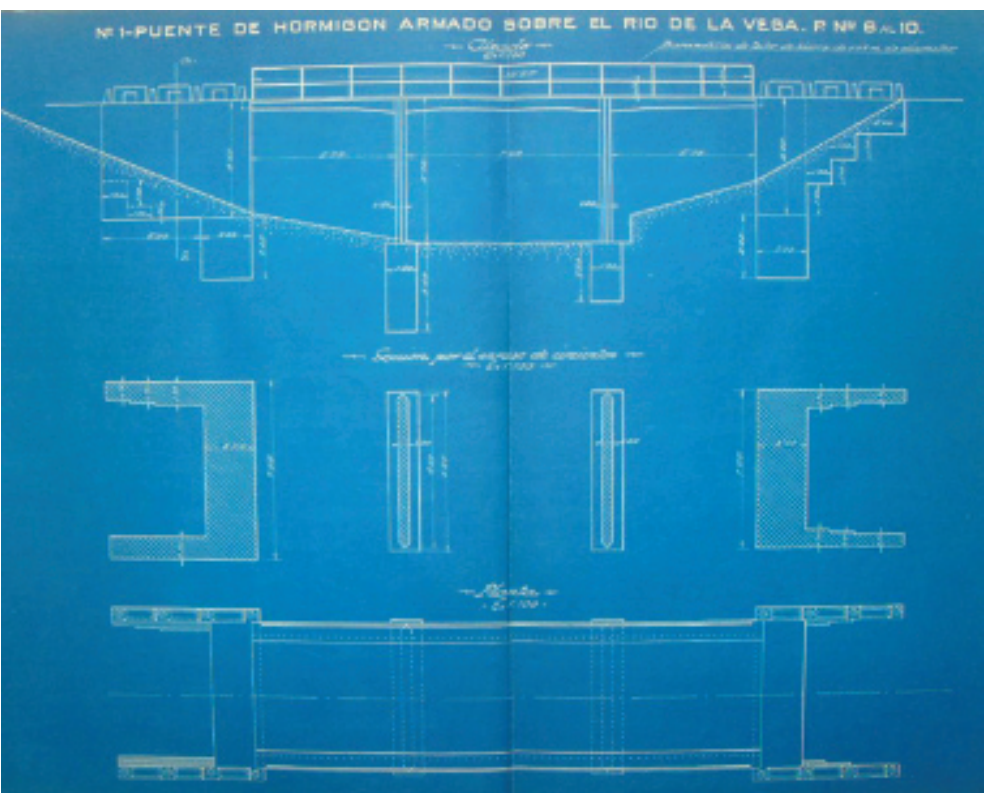

Esta infraestructura incorporaba dos puentes de cierta envergadura: uno sobre el río Guadalimar y otro sobre su afluente el Onsares. En esta ocasión, Acuña los proyectó conforme a los modelos de la "Colección" oficial, a base de tramos rectos de hormigón armado con sección transversal en $\pi$ (Figura 7).

$\mathrm{Al}$ igual que en el caso anterior, estos puentes fueron formulados conforme al conjunto de tipologías descritas por Fernández Casado en (20) y (21).

En el caso concreto de la propuesta de Fernández Casado para el torrente del Onsares, mejoraba funcional y económicamente
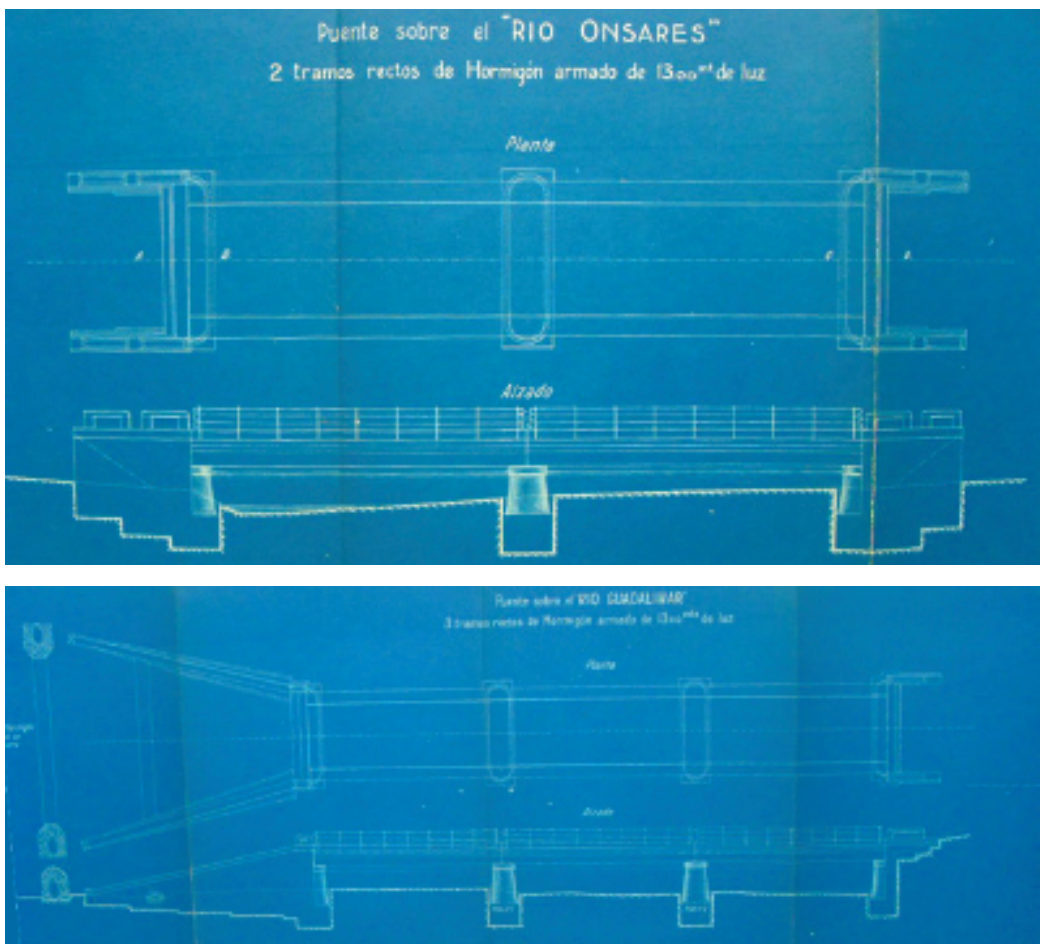

5. Puente sobre el río de la Vega en Santo Tomé en la carretera de 3er orden de Peal de Becerro a Villacarrillo. 1934. Plano de alzado general (22)

6. Construcción del puente sobre el río de la Vega en Santo Tomé. 1933 (24).

7. Proyecto de carretera de 3 er orden de la Puerta de Segura a Siles. 1929. Puentes sobre e Onsares y el Guadalimar. Planos de alzado general (26). 

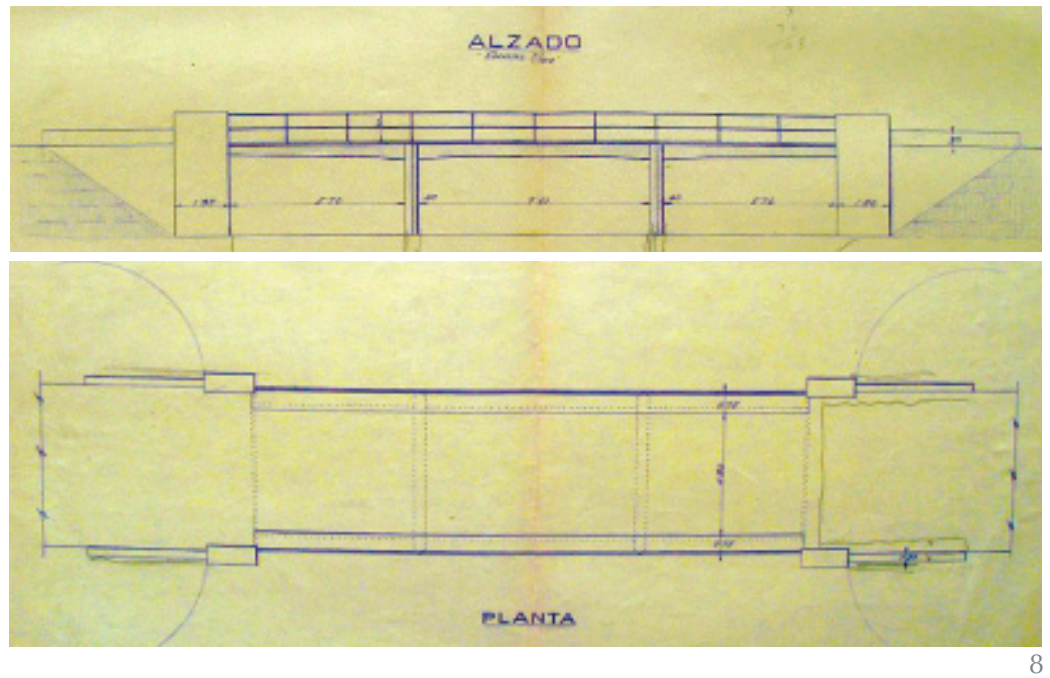

8. Puente sobre el río Onsares. Carretera de 3er orden de La Puerta a Siles. 1934. Plano de alzado y planta para cubicación (27).

9. Puente sobre el río Onsares. Carretera de 3 er orden de La Puerta a Siles. 1934 (28).

10.NPuente sobre el río Guadalimar. Carretera de 3er orden de La Puerta a Siles. 1934 (29). el proyecto de Acuña. Se trataba de otro pórtico en $\pi$ de tres vanos con tablero de losa (serie III), con las mismas luces $(5,70$ 7,60-5,70m) que el puente sobre el río de la Vega. Se solucionaba así el problema con un puente más liviano y que no alteraba la rasante de la carretera, pero en cambio con sección de desagüe muyo mayor, según puede verse en la Figura 8.

De todos los puentes de altura estricta que se construyeron, éste podría ser uno de los más representativos del concepto estético de Fernández Casado, explícitamente destacado en la justificación de la "Colección" como uno de sus principales objetivos

Como se observa en la Figura 9, este puente, pegado al horizonte (tenía sólo 2,50 m de altura de rasante), con su finísimo tablero y sus delgadas pilas, refleja con total fidelidad la idea de la altura estricta. Por ello su desaparición (ha sido sustituido recientemente por un impersonal puente de vigas pretensadas), constituye un hecho especialmente lamentable.

Para el otro puente importante de esta carretera, el del Guadalimar (Figura 10), Fernández Casado, si bien mantuvo la configuración de pórtico en $\pi$ de tres vanos, se vio obligado a recurrir a la opción de tablero con vigas para poder alcanzar una longitud total mayor, del orden de 40 metros (luces 12-16-12m).

En este primer puente de vigas el diseño todavía no estaba completamente depurado, presentando acartelamientos más reducidos que en los puentes posteriores (30). De ahí que tenga una apariencia más robusta.

\subsection{Pasos superiores y Puente sobre el río Guadalimar en la Puerta de Segura. 1935}

Estos tres puentes, los primeros de la "Colección" estaban terminados a principios de
1934. Después, Carlos Fernández Casado, ya definitivamente instalado en Madrid, se dedicó enteramente al proyecto y construcción de sus famosos puentes de El Pardo y de Puerta de Hierro. No obstante, hasta el estallido de la Guerra Civil seguiría colaborando con José Acuña en la construcción de otros tres puentes de altura estricta de la provincia de Jaén. Respondiendo a necesidades diferentes, presentarían tipologías variadas: dos pequeños pasos superiores sobre arroyos en caminos vecinales y un tercero, de mayor entidad sobre el río Guadalimar en la Puerta de Segura.

Los pasos superiores se situarían en los caminos vecinales de Hortizuela a Bienservida y de Orcera a Hornos. En cuanto a sus características estructurales ambos casos se solucionaron mediante pórtico sencillo (serie I) con dintel de losa de $30 \mathrm{~cm}$ de canto y $8,00 \mathrm{~m}$ de luz. En palabras del propio Fernández Casado eran "obras de construcción muy sencilla y que quedaban en perfectas condiciones de sumergibilidad" (31).

Por su parte el puente sobre el río Guadalimar en la Puerta de Segura cerró la cola-
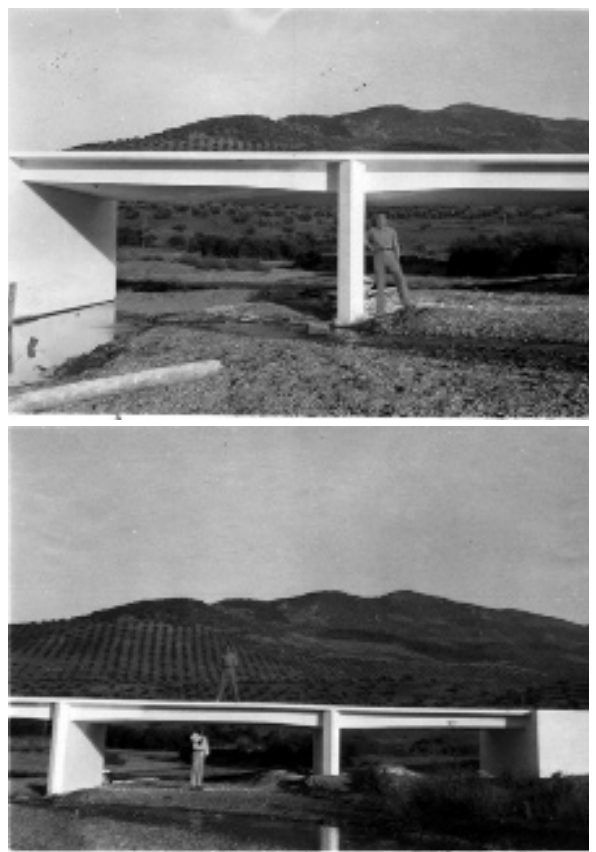

9

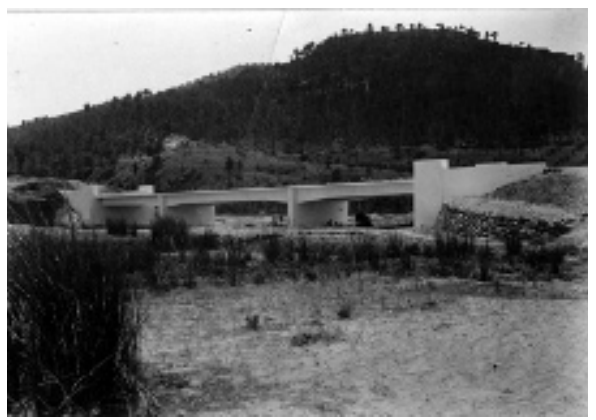

10 
boración entre Fernández Casado y Acuña. Fue diseñado para la carretera que une esta última localidad con la de Puente Génave.

A diferencia de los anteriores la estructura de este puente esta resuelta en cinco tramos de luces 6,50-9,00-9,00-9,00-6,50 m, alcanzando una longitud total de $40 \mathrm{~m}$. El tablero es de losa, con el vano central en cantiléver.

Esta configuración (una adaptación de la serie VII) fue poco utilizada, por lo que se trata ciertamente de uno de los puentes de altura estricta más singulares.

Una imagen del puente poco después de su construcción se muestra en la Figura 11.

\subsection{Los puentes en la actualidad}

De los seis puentes resultantes de la colaboración entre Carlos Fernández Casado y José Acuña sólo quedan en la actualidad tres. Sobreviven en general en buen estado, pese a encontrarse en entornos muy degradados y faltos de todo cuidado por parte de las administraciones competentes.

Su gran valor histórico y patrimonial no está reconocido, pasando su existencia totalmente desapercibida, a pesar de que dos de ellos se encuentran prácticamente en entornos urbanos.

El puente sobre el río de la Vega en Santo Tomé continúa en uso y casi intacto, prueba de sus magníficos diseño y ejecución (Figu-
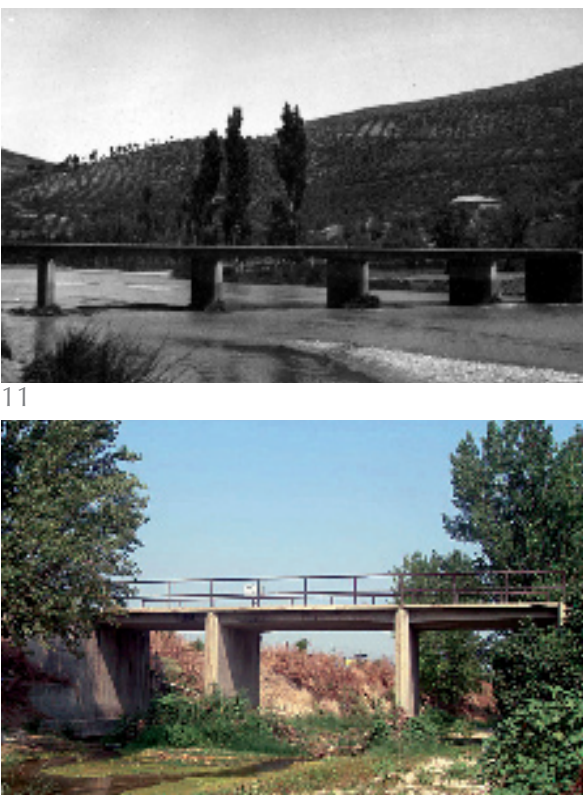

ras 5 y 12). No obstante, aguas abajo se han añadido unas soleras de encauzamiento del arroyo y una rudimentaria estación de aforos que distorsionan en parte su imagen.

De los dos puentes de la carretera de La Puerta de Segura a Siles sólo permanece en pie el del río Guadalimar (Figuras 13a y 13b). El del Onsares, tal y como se ha comentado, ha desaparecido en una reciente remodelación de la carretera. Afortunadamente, en esta misma actuación se decidió conservar el del río Guadalimar, dejándolo en un ramal aislado y conduciendo el tráfico por un puente nuevo tipo estándar.
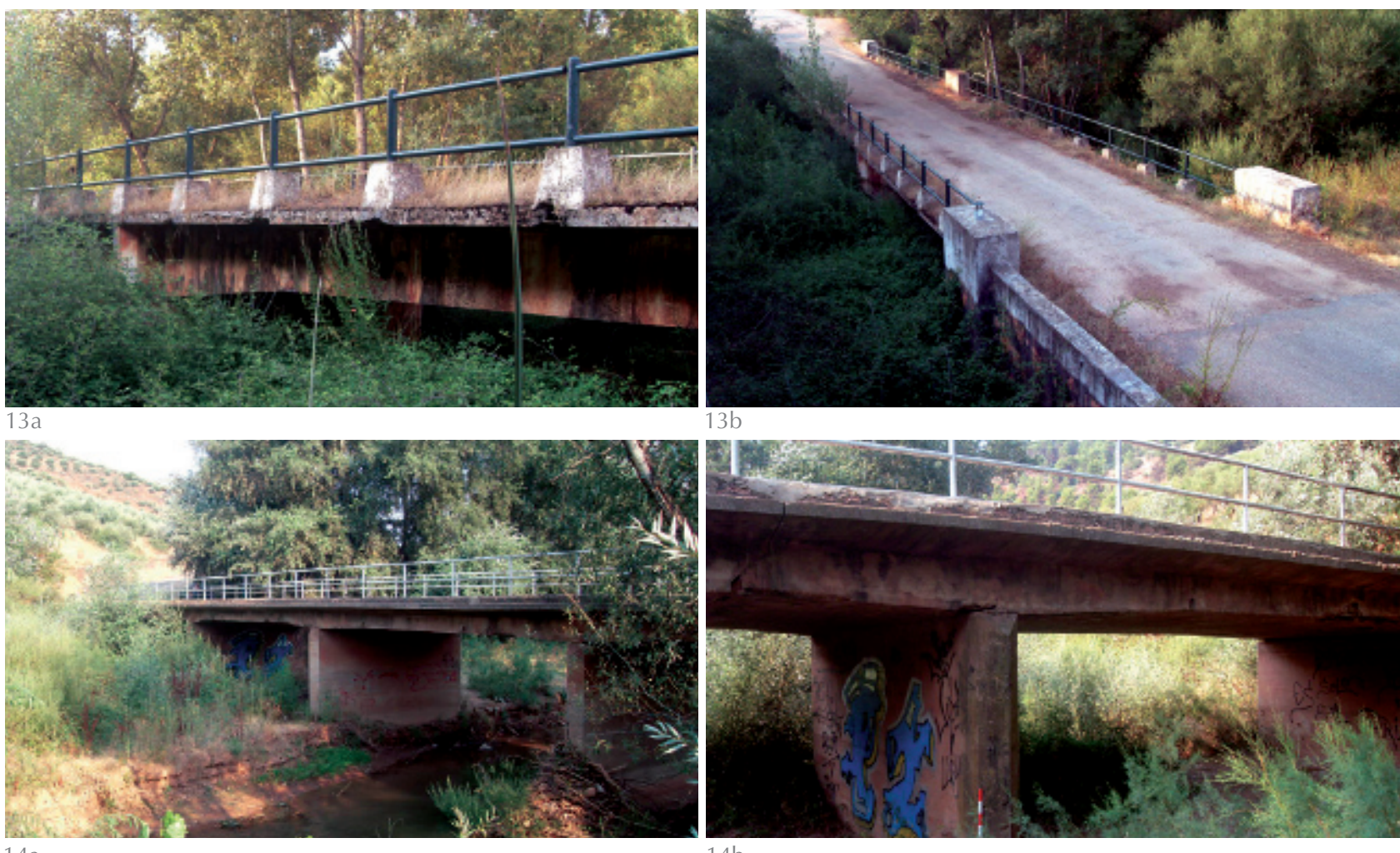
15. Configuraciones estructurales de los puentes de Altura Estricta de la provincia de Jaén (33).
Como se puede observar en las figuras precedentes, el puente está oculto entre una abundante vegetación, que dificulta su reconocimiento en conjunto y la visión de sus elementos estructurales.

El tercer puente que se conserva también salva el río Guadalimar, esta vez inmediatamente aguas abajo de la localidad de La Puerta de Segura (en cuyo entorno urbano está prácticamente integrado). Por esta circunstancia ofrece buenas posibilidades de reutilización, siempre y cuando se acondicione la zona circundante al emplazamiento (Figura 14).

Actualmente sigue en uso, formando parte de la carretera para la que se proyectó. Por su mayor complejidad estructural (comentada en apartado anterior) requiere mayores cuidados, lo que en su ausencia ha provocado que se encuentre en peor estado que los anteriores. No obstante, su estado de deterioro no es irreversible.

En esta última figura se observa con detalle la pila y la articulación con la que se forma el tramo central en cantiléver.

\section{RESULTADOS}

A partir de la documentación a la que se ha tenido acceso y la información recabada "in situ" en las distintas localizaciones se comentan las características más relevantes de cada puente. Se resumen en la siguiente Tabla 1.

En la siguiente figura (Figura 15) se recogen los croquis originales que realizó Carlos Fernández Casado con las configuraciones estructurales de los puentes de Jaén.

En cuanto a sus características constructivas y funcionales se constata mediante la documentación analizada y la observación de los puentes que las premisas básicas (descritas con carácter general en el apartado la "Colección de puentes de altura estricta". Aspectos constructivos y funcionales. Premisas básicas del diseño) a partir de las cuales el autor justificaba la idoneidad de su diseño se satisfacen en los puentes de Jaén,

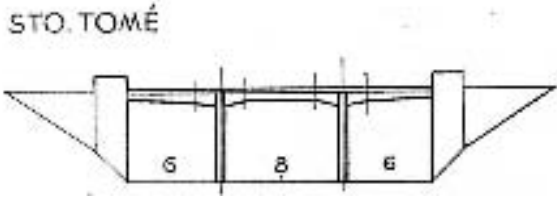

ONSARES
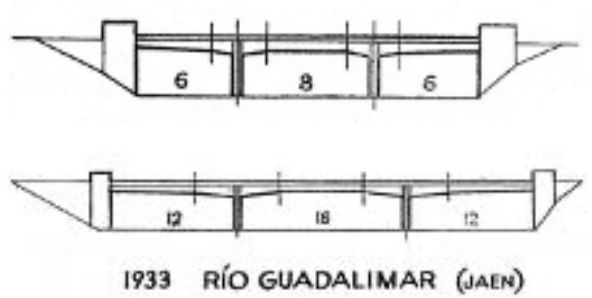

PUERTA DEL SEGURA

PUERTA

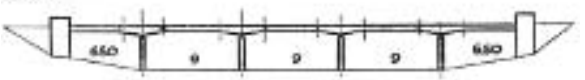

15

a pesar de tratarse de las primeras aplicaciones con todo lo que ello conllevaba.

De tal forma que:

- La tipología estructural es la más apropiada en cada caso desde el punto de vista técnico y económico.

- El sistema de ejecución era de gran sencillez.

- La disposición de los puentes reducía notablemente el coste de la infraestructura, eliminando muros de acompañamiento y minimizando el volumen de material de aportación en estribos.

- Se garantizaba una sección de desagüe suficiente en cualquier circunstancia.

Por último todo lo anterior se obtenía sin menoscabo de una estética cuidada que por si misma constituía uno de los objetivos básicos perseguidos con el diseño de los puentes.

\section{CONCLUSIONES}

En la provincia de Jaén se conservan algunas de las materializaciones más representativas de la "Colección de puentes de altura estricta" de Carlos Fernández Casado,

Tabla 1. Resumen de características de los puentes de altura estricta en Jaén, 1933-1935.

\begin{tabular}{|c|c|c|c|c|c|c|}
\hline \multirow{2}{*}{ Denominación } & \multirow{2}{*}{ Año } & \multicolumn{4}{|c|}{ Configuración estructural } & \multirow{2}{*}{$\begin{array}{l}\text { Existe en la } \\
\text { actualidad }\end{array}$} \\
\hline & & Tablero & Tipología & Luz (m) & Uso & \\
\hline Río de la Vega & 1933 & Losa & 3 vanos & 20 & Carretera & $\mathrm{SI}$ \\
\hline Onsares & 1934 & Losa & 3 vanos & 20 & Carretera & $\mathrm{NO}$ \\
\hline Guadalimar-Crta. Siles & 1934 & Vigas & 3 vanos & 40 & Carretera & $\mathrm{SI}$ \\
\hline Paso superior Hortizuela & 1935 & Losa & 1 vano & 8 & Camino vecinal & $\mathrm{NO}$ \\
\hline Paso superior Orcera & 1935 & Losa & 1 vano & 8 & Camino vecinal & $\mathrm{NO}$ \\
\hline $\begin{array}{l}\text { Guadalimar } \\
\text { La Puerta de Segura }\end{array}$ & 1935 & Losa & $\begin{array}{l}5 \text { vanos } \\
\text { cantiléver }\end{array}$ & 40 & Carretera & SI \\
\hline
\end{tabular}


una de las creaciones trascendentales de la Ingeniería Civil española del siglo XX.

Constituyen una muestra muy representativa de las posibilidades de aplicación de la "Colección", dando en todos los casos una respuesta satisfactoria a las necesidades concretas de cada emplazamiento, a partir de un diseño idóneo correctamente formulado de acuerdo con los postulados definidos por el autor.

Desgraciadamente sólo tres de ellos se conservan en la actualidad. Se trata de puentes de gran valor, en primer lugar en sí mismos como obras de ingeniería, por su diseño estructural y funcional, por su perfecta integración en el lugar donde se ubican y por su extraordinaria y original estética.
Desde el punto de vista de la historia de la construcción también tienen gran importancia, pues ya con más de tres cuartos de siglo en servicio se han convertido en elementos patrimoniales, testimonios de la mejor ingeniería de su tiempo.

Prácticamente ignorada hasta hoy su existencia, y desconocida de forma generalizada su importancia, corren peligro de desaparecer, como de hecho ha ocurrido ya con alguno de los puentes.

Se espera que este artículo pueda contribuir a paliar este desconocimiento, esperando estimule a las autoridades competentes a emprender las actuaciones necesarias para su puesta en valor y preservación.

\section{BIBLIOGRAFIA}

(1) Fernández Casado, C.: "Puentes de altura estricta. Realizaciones desde 1933 a 1955". Informes de la Construcción, $\mathrm{n}^{\circ} 72$ separata (1956), p.31.

(2) Sáenz Ridruejo, F.: "Sánchez del Río y Fernández Casado, dos ingenieros de la generación del 27". Actas del VIII Congreso de la Sociedad Española de Historia de las Ciencias y de las Técnicas. p.156, Universidad de la Rioja. Logroño, 2004.

(3) Proyecto de sustitución del paso a nivel de Mengibar en el camino nacional 323 de Bailén a Motril, km 309,71 en su cruce con el ferrocarril de Puente Genil a Linares, km 147,757. Archivo General de la Administración. Signatura 24/13492.

(4) Fernández Casado, C.: "Colección de puentes de altura estricta". Revista de Obras Públicas, año LXXXII, nº 2637 (1934).

(5) Cuéllar, D.: Los transportes en el Sureste Andaluz (1850-1950): Economía, Empresas y Territorio. pp. 116-119, Fundación de los Ferrocarriles Españoles. Madrid, 2003.

(6) Orden Ministerial 07/06/1919. Gaceta de Madrid, nº158 (1919), Ministerio de Fomento, Dirección General de Obras Públicas. Madrid.

(7) Del Cuvillo, R.: Colecciones oficiales de obras de paso de carreteras (siglos XIX y XX). pp. 15-60, Colegio de ingenieros de Caminos, Canales y Puertos. Madrid, 2007.

(8) Benet, J.: "Carta para la presentación del libro Ingeniería Hidráulica Romana". Boletín de información del colegio de ingenieros de Caminos, Canales y Puertos, n 158 (1985), p.22.

(9) Fernández Troyano, L.; et ál.: Carlos Fernández Casado, Ingeniero. pp. 30-32, Ministerio de Fomento CEHOPU-CEDEX. Madrid, 2007.

(10) Fernández Casado, C.: "Colección de puentes de altura estricta". Revista de Obras Públicas, nº 2701 (1936), p.1.

(11) Fernández Casado, C.: "Puentes de altura estricta. Realizaciones desde 1933 a 1955". Informes de la Construcción, no 72 separata (1956), Lámina 1.

(12) Fernández Casado, C.: "Colección de puentes de altura estricta. El método de Cross para el cálculo de entramados". Revista de Obras Públicas, nº 2642 (1934), p.1.

(13) Archivo Carlos Fernández Casado, CEHOPU-CEDEX. FC-006.

(14) Fernández Casado, C.: "Puentes de altura estricta. Realizaciones desde 1933 a 1955". Informes de la Construcción, $\mathrm{n}^{\circ} 72$ separata (1956), p.1.

(15) "Caminos vecinales en la provincia de Jaén". ABC, 24-08-1927, p.23.

(16) Acuña, J.: Replanteo previo de los trozos $3^{\circ}$ y $4^{\circ}$ de la carretera de tercer orden de Peal de Becerro a Villacarrillo. 1932. Archivo Histórico Provincial de Jaén, nº 31447.

(17) Carta de los miembros de la comisión gestora del Ayuntamiento de Peal de Becerro al Ministro de Fomento, 23 de junio de 1931. Archivo Histórico Provincial de Jaén, $\mathrm{n}^{\circ}$ 31447.

(18) Fernández Casado, C.: Proyecto de aprovechamiento hidráulico del río Susana. 1930. Archivo de la Comisaría de Aguas de Sevilla, n 643.

(19) Acuña, J.: Proyecto de puente sobre el río de la Vega en Santo Tomé para la carretera de 3er orden de Peal de Becerro a Villacarrillo. 1932. Archivo histórico provincial de Jaén nº 31447.

(20) Fernández Casado, C.: "Colección de puentes de altura estricta", Revista de Obras Públicas, año LXXXIV, nº 2701. Madrid, (1936). 
(21) Fernández Casado, C.: "Puentes de altura estricta. Realizaciones desde 1933 a 1955". Informes de la Construcción, $\mathrm{n}^{\circ} 72$ separata (1956), p.1. Referencia al Ministerio de obras públicas. 1942. Declaración de Colección Oficial.

(22) Fernández Casado, C.: Liquidación de las obras de fábrica de la carretera de 3er orden de Peal de Becerro a Villacarrillo. 1934. Archivo Histórico Provincial de Jaén no 31447.

(23) Acta de replanteo definitiva de las obras construidas en el 3er trozo de la carretera de 3er orden de Peal de Becerro a Villacarrillo, 12 de enero de 1936. Archivo Histórico Provincial de Jaén, $n^{\circ} 31447$.

(24) Archivo Carlos Fernández Casado. CEHOPU-CEDEX. FC-001.

(25) Acuña, J.: Proyecto de carretera de 3er orden de La Puerta a Siles. 20 de diciembre de 1929. Archivo Histórico Provincial de Jaén, nº 31502.

(26) Acuña, J.: Proyecto de carretera de 3er orden de la Puerta de Segura a Siles. 1929. Puentes sobre el Onsares y el Guadalimar. Archivo Histórico Provincial de Jaén $n^{\circ}$ 31502.

(27) Fernández Casado, C.: Puente sobre el río Onsares. Carretera de 3er orden de La Puerta a Siles. 1934. Archivo Histórico Provincial de Jaén nº 31503.

(28) Archivo Carlos Fernández Casado, CEHOPU-CEDEX. I/FC-002/001.

(29) Archivo Carlos Fernández Casado, CEHOPU-CEDEX. I/FC-005/001.

(30) Fernández Casado, C.: "Puentes de altura estricta. Realizaciones desde 1933 a 1955". Informes de la Construcción, $\mathrm{n}^{\circ} 72$ separata (1956), p.2.

(31) Fernández Casado, C.: "Colección de puentes de altura estricta". Revista de Obras Públicas, $\mathrm{n}^{\circ} 2701$ (1936), p.7.

(32) Archivo Carlos Fernández Casado, CEHOPU-CEDEX. FC-006.

(33) Fernández Casado, C.: "Puentes de altura estricta. Realizaciones desde 1933 a 1955". Informes de la Construcción, nº 72 separata (1956), Lámina XVI y XVII. 